\title{
Association of Somatic Symptom Severity With Sociodemographic Parameters In Patients With Medically Unexplained Physical Symptoms: A Cross-Sectional Study From a Tertiary Care Center in India
}

\author{
Upendra Baitha ${ }^{1}$, Piyush Ranjan ${ }^{1}$, Koushik Sinha Deb ${ }^{2}$, Nitesh K. Bauddh ${ }^{3}$, Vishwajeet Singh ${ }^{4}$, \\ Gaurishanker Kaloiya ${ }^{5}$, Arvind Kumar ${ }^{1}$, Anamika Sahu ${ }^{2}$ \\ 1. Medicine, All India Institute of Medical Sciences, Delhi, IND 2. Psychiatry, All India Institute of Medical Sciences, \\ Delhi, IND 3. Medicine, Government Medical College, Kota, IND 4. Biostatistics, All India Institute of Medical Sciences, \\ Delhi, IND 5. Clinical Psychology, All India Institute of Medical Sciences, Delhi, IND
}

Corresponding author: Piyush Ranjan, drpiyushdost@gmail.com

\section{Abstract}

\section{Background}

There is a paucity of studies assessing the severity of somatic symptoms in medically unexplained physical symptoms (MUPS) from Medicine outpatient department (OPD).

\section{Methodology}

This cross-sectional study was conducted in Medicine OPD of a tertiary care hospital in India, in which 245 MUPS-diagnosed patients out of 976 consecutive screened patients were evaluated for the severity of somatic symptoms (by administering the Patient Health Questionnaire-15) and its sociodemographic correlates.

\section{Results}

Out of 245 recruited patients, three-fourth had a significant severity level of somatic symptoms. High level of somatic symptom severity was more common in females $(p \leqslant 0.001)$, married patients $(p=0.011)$, rural dwellers $(p=0.035)$, less educated $(p=0.003)$, and those with lower socioeconomic status $(p=0.001)$.

\section{Conclusions}

Patients with MUPS have a high level of somatic symptom severity with certain sociodemographic correlates. Further research should be conducted to investigate the reasons for this and to formulate a costeffective treatment strategy.

Received 06/25/2020

Review began 06/29/2020 Review ended 07/04/2020 Published 07/17/2020

(c) Copyright 2020

Baitha et al. This is an open access article distributed under the terms of the Creative Commons Attribution License CC-BY 4.0., which permits unrestricted use, distribution, and reproduction in any medium, provided the original author and source are credited.
Categories: Internal Medicine, Psychiatry

Keywords: medically unexplained physical symptoms, somatic symptoms, correlates, india

\section{Introduction}

Patients with medically unexplained physical symptoms (MUPS) are characterized by persistent somatic complaints for which adequate clinical examination and relevant investigations do not reveal sufficient explanation by known disease. These patients present with multiple somatic symptoms such as pain, fatigue, functional disturbances, and other systemic complaints such as neurological and gastrointestinal complaints.

They constitute a considerable portion of patients in all levels of health care, particularly in general care settings where prevalence rates are reported between $20 \%$ and $30 \%$ [1,2]. Typically, these patients seek multiple consultations from different specialties and utilize more health care services, leading to a substantial burden on health care systems [3]. Studies suggest that these patients have significant disabilities and poor quality of life, which may be due to the high burden of somatic symptoms $[4,5]$.

Studies assessing the severity of somatic symptoms in patients with MUPS from the Medical outpatient department (OPD) facilities are scarce. One such study with a small sample size suggests that the severity of somatic symptoms is more in patients with MUPS than in patients with medically explained symptoms [6]. The sociodemographic correlates of the severity of somatic symptoms in MUPS have not been studied elaborately. We conducted this cross-sectional study to assess the severity of somatic symptoms by administering the Patient Health Questionnaire-15 (PHQ-15) among patients with MUPS in a tertiary care 
hospital of India and evaluated the sociodemographic factors affecting the severity of the symptoms in these patients.

\section{Materials And Methods}

This cross-sectional observational study was conducted in the Medicine OPD of a tertiary care hospital in North India. The Institution Ethics Committee approved the study, and written consent was taken from all patients recruited in the study. A total of 976 consecutive patients presenting to the designated OPD rooms of Medicine department, over a period of one year were assessed for MUPS and were recruited according to the inclusion and exclusion criteria. For this study, MUPS was defined as any current principal symptom(s) in a patient for which no medical diagnosis could be reached by detailed clinical examination and appropriate investigations [7]. Each case was reviewed by two independent physicians before establishing a diagnosis of MUPS. Referrals from other subspecialties were also taken. Patient's data regarding sociodemographic correlates such as age, gender, marital status, educational level, employment status, and occupation were recorded in pre-validated preform that was developed specifically for the index study. Assessment of the severity of the symptoms was performed using PHQ-15 [8,9]. PHQ-15 is a validated 15item questionnaire that assesses the severity of somatic symptoms. Each item asks the participant to rate the severity of the somatic symptom experienced during the past seven days on a 3-point scale ( 0 if not bothered at all, 1 if bothered a little, and 2 if the symptom bothered a lot). The total score varies from 0 to 30, with higher scores signifying greater severity of somatic symptoms. The level of somatic symptom severity is interpreted as minimal, low, moderate, and high for a PHQ-15 score of 0-4, 5-9, 10-14, and 15-30, respectively.

Each qualitative variable was expressed as an absolute and relative frequency, whereas the continuous variable was expressed as mean along with standard deviation. To find the association between qualitative variables, the chi-square test or Fisher exact test was used according to the distribution of data. A p-value of less than 0.05 was considered statistically significant.

\section{Results}

A total of 976 consecutive patients who presented to the designated OPD rooms of the Medicine Department were assessed for the presence of MUPS. Among them, 245 patients were diagnosed to have MUPS and were subsequently recruited in the study. Most of the patients included in the study were young, married, and living in a joint family. The majority of them belonged to the middle socioeconomic class and have completed school education. Sixty-one (25\%) patients had a minimal/low level of severity of somatic symptoms, whereas 105 (46\%) and 79 (29\%) patients had medium and high levels of severity of somatic symptoms, respectively. Their sociodemographic parameters, levels of severity of somatic symptoms, and an association between the two are included in Table 1. 


\section{Cureus}

\begin{tabular}{|c|c|c|c|c|c|}
\hline \multirow{3}{*}{ Variables } & \multicolumn{4}{|c|}{ Numbers of patients with MUPS } & \multirow{3}{*}{ p-Value } \\
\hline & \multirow{2}{*}{ Total $(n=245)$} & \multicolumn{3}{|c|}{ Levels of the severity of symptoms } & \\
\hline & & Minimal/low $(n=61)$ & Medium $(n=105)$ & High $(n=79)$ & \\
\hline Age (in years) & & & & & \\
\hline Less than 30 years & $86(35 \%)$ & $28(46 \%)$ & 37 (35\%) & $21(26 \%)$ & \multirow{4}{*}{0.185} \\
\hline $30-40$ years & $91(37 \%)$ & $23(38 \%)$ & $36(34 \%)$ & $32(40 \%)$ & \\
\hline $40-50$ years & $49(20 \%)$ & $06(10 \%)$ & $23(22 \%)$ & $20(25 \%)$ & \\
\hline More than 50 years & $19(8 \%)$ & $04(6 \%)$ & $09(9 \%)$ & $06(9 \%)$ & \\
\hline \multicolumn{6}{|l|}{ Gender } \\
\hline Male & $107(44 \%)$ & $40(66 \%)$ & $48(46 \%)$ & $19(24 \%)$ & \multirow{2}{*}{$<0.001$} \\
\hline Female & $138(56 \%)$ & $21(34 \%)$ & $57(54 \%)$ & 60 (76\%) & \\
\hline \multicolumn{6}{|l|}{ Marital status } \\
\hline Married & $176(72 \%)$ & $34(56 \%)$ & 79 (75\%) & $63(80 \%)$ & \multirow{3}{*}{0.011} \\
\hline Single & $62(25 \%)$ & $25(41 \%)$ & $24(23 \%)$ & $13(16 \%)$ & \\
\hline Other & $7(3 \%)$ & $2(3 \%)$ & $2(2 \%)$ & $3(4 \%)$ & \\
\hline \multicolumn{6}{|l|}{ Locality } \\
\hline Urban & $138(56 \%)$ & $41(67 \%)$ & $61(58 \%)$ & $36(46 \%)$ & \multirow{2}{*}{0.034} \\
\hline Rural & 107 (44\%) & $20(33 \%)$ & $44(42 \%)$ & $43(54 \%)$ & \\
\hline \multicolumn{6}{|l|}{ Family type } \\
\hline Residing & $18(7 \%)$ & $06(10 \%)$ & $09(9 \%)$ & $03(4 \%)$ & \multirow{3}{*}{0.544} \\
\hline Alone & $85(30 \%)$ & $23(38 \%)$ & $3(31 \%)$ & $29(37 \%)$ & \\
\hline Nuclear joint & $142(58 \%)$ & $32(52 \%)$ & $63(60 \%)$ & $47(59 \%)$ & \\
\hline \multicolumn{6}{|l|}{ Socioeconomic class } \\
\hline Upper & $22(09 \%)$ & $11(18 \%)$ & $10(9 \%)$ & $01(1 \%)$ & \multirow{5}{*}{0.001} \\
\hline Upper middle & $51(21 \%)$ & $17(28 \%)$ & $25(24 \%)$ & $09(11 \%)$ & \\
\hline Lower middle & $58(23 \%)$ & $13(21 \%)$ & $26(25 \%)$ & $19(24 \%)$ & \\
\hline Upper lower & $95(39 \%)$ & $19(31 \%)$ & $36(34 \%)$ & $40(51 \%)$ & \\
\hline Lower & $19(8 \%)$ & $01(02 \%)$ & $08(8 \%)$ & $10(13 \%)$ & \\
\hline IIIIterate & $57(23 \%)$ & $08(13 \%)$ & $22(21 \%)$ & $26(33 \%)$ & \multirow{4}{*}{0.003} \\
\hline Primary school & $45(18 \%)$ & $08(13 \%)$ & $18(17 \%)$ & $19(24 \%)$ & \\
\hline Secondary & 79 (32\%) & 19 (31\%) & 37 (35\%) & 23 (29\%) & \\
\hline School graduate and above & $65(25 \%)$ & $26(43 \%)$ & $28(27 \%)$ & $11(14 \%)$ & \\
\hline
\end{tabular}

TABLE 1: Association between symptoms severity and sociodemographic parameters

\section{Discussion}

The study of somatic symptom burden is of paramount importance in any disease. It is directly related to the sense of well-being, quality of life, and health care expenses [10,11]. The study of somatic symptom burden has attracted the interests of researchers from different parts of the world and researchers have studied it in 
common clinical conditions such as anxiety, depression, chronic obstructive pulmonary disease (COPD), and cardiac illnesses [12-14]. In a large study involving 1,329 patients with anxiety and depression disorders, it has been found that $72.2 \%, 20.7 \%$, and $7.1 \%$ of patients had a minimal/mild, medium, and high level of somatic symptom severity, respectively [12]. In another study involving COPD patients, it was found that minimal/mild, medium, and high level of somatic symptom severity was present in $45.5 \%, 27.3 \%$, and $27.3 \%$ of the patients studied, respectively [13]. In comparison to these common conditions, patients with MUPS have a notably high level of somatic symptom severity. Three-fourth of the consecutive patients of MUPS enrolled in our study had significant (medium or high) levels of the severity of somatic symptoms, of which one-third of the patients had high levels of somatic symptom severity. These findings are in accordance with a cross-sectional retrospective study conducted on 294 primary care patients with MUPS in Germany, which revealed that $29 \%, 37 \%$, and $34 \%$ of the MUPS patients had low, medium, and high levels of somatic symptom severity, respectively [3].

The sociodemographic evaluation of the findings of the index study suggests that a high level of somatic symptom severity is associated with female gender $(\mathrm{p}<0.001)$ and married status $(\mathrm{p}=0.011)$. This finding can be attributed to women's tendency to perceive physical sensations as more bothersome and seek more frequent medical help in comparison to their male counterparts $[15,16]$. Women are emotionally more responsive and expressive than men and thus their somatic complaints are more likely to be evaluated and reported [17]. It has been reported that married and widowed women are at higher risk of developing MUPS than single women because of the emotional and social stresses that these women are subjected to, and these emotions get expressed through physical symptoms that are acceptable to the society [18]. Besides, incidences of past abuses and trauma are more common in females [19].

In our study, patients with lower socioeconomic status and less education had a more likelihood of developing high levels of somatic symptom severity. Studies have been conducted to establish a relationship between socioeconomic status, education status, and common mental disorders. Although most of these studies establish a positive correlation between lower socioeconomic status and poor mental health outcomes, some have reported contrary findings [20-22]. Education is supposed to enhance people's skills and empower them with a better coping mechanism, leading to better mental health [23].

This study is one of the initial large studies that evaluated levels of severity of somatic symptoms in patients with MUPS. MUPS is a common yet neglected clinical entity in this part of the world. The patients with MUPS have higher somatic symptom severity than many other common clinical conditions and have interesting sociodemographic correlates that have not been studied elaborately yet. This study has found that MUPS patients who are females, married, residing in rural areas, less educated, and with lower socioeconomic status are more likely to have a severe level of somatic symptoms. The prevalence of coexisting physical and psychological co-morbidities and their impact on symptom severity in MUPS patients have not been assessed in this study but can be explored in future research. Also, newer treatment modalities such as yoga can be explored that may be useful in reducing somatic symptom severity, quality of life, and associated psychiatric comorbidities [24]. Multidisciplinary treatment modalities such as an MUPS clinic should be opened in association with psychiatrists and psychologists.

This being a cross-sectional study, the causal association and odds ratio cannot be measured, and this is a limitation of the study. Cohort studies should be conducted to establish a causal association.

\section{Conclusions}

Patients with MUPS have a considerable level of somatic symptom severity. MUPS patients with female gender, married status, rural residence, lower socioeconomic status, and less education level have a high level of somatic symptom severity.

\section{Additional Information \\ Disclosures}

Human subjects: Consent was obtained by all participants in this study. Institute Ethics Committee, All India Institute of Medical Sciences, New Delhi issued approval IEC/750/9/2019. Animal subjects: All authors have confirmed that this study did not involve animal subjects or tissue. Conflicts of interest: In compliance with the ICMJE uniform disclosure form, all authors declare the following: Payment/services info: All authors have declared that no financial support was received from any organization for the submitted work. Financial relationships: All authors have declared that they have no financial relationships at present or within the previous three years with any organizations that might have an interest in the submitted work. Other relationships: All authors have declared that there are no other relationships or activities that could appear to have influenced the submitted work.

\section{References}

1. Baitha U, Deb KS, Ranjan P, et al.: Estimated prevalence of medically unexplained physical symptoms in the medicine outpatient department of a tertiary care hospital in India. Gen Hosp Psychiatry. 2019, 61:47-52. 10.1016/j.genhosppsych.2019.10.006 
2. Hilderink PH, Collard R, Rosmalen JGM, Oude Voshaar RC: Prevalence of somatoform disorders and medically unexplained symptoms in old age populations in comparison with younger age groups: a systematic review. Ageing Res Rev. 2013, 12:151-6. 10.1016/j.arr.2012.04.004

3. Konnopka A, Kaufmann C, König H-H, et al.: Association of costs with somatic symptom severity in patients with medically unexplained symptoms. J Psychosom Res. 2013, 75:370-5. 10.1016/j.jpsychores.2013.08.011

4. Kushwaha V, Sinha Deb K, Chadda RK, Mehta M: A study of disability and its correlates in somatisation disorder. Asian J Psychiatry. 2014, 8:56-8. 10.1016/j.ajp.2013.10.016

5. Rao GR, Ranjan P, Chadda RK, et al.: A descriptive study of disability in patients presenting with medically unexplained physical symptoms in a medical OPD setting. J Family Med Prim Care. 2019, 8:1755-9. 10.4103/jfmpc.jfmpc_223_19

6. Sharma M, Singh SM, Avasthi A, Varma SC, Sharma A, Suri V: Medically unexplained physical symptoms in patients attending a medical outpatient clinic in a tertiary hospital in North India. Asian J Psychiatry. 2018, 32:99-104. 10.1016/j.ajp.2017.11.022

7. Hatcher S, Arroll B: Assessment and management of medically unexplained symptoms . BMJ. 2008, 336:1124-8. 10.1136/bmj.39554.592014.BE

8. Kroenke K, Spitzer RL, Williams JBW: The PHQ- 15: validity of a new measure for evaluating the severity of somatic symptoms. Psychosom Med. 2002, 64:258-66. 10.1097/00006842-200203000-00008

9. Kocalevent R-D, Hinz A, Brähler E: Standardization of a screening instrument (PHQ-15) for somatisation syndromes in the general population. BMC Psychiatry. 2013, 13:91. 10.1186/1471-244X-13-91

10. Kliem S, Lohmann A, Klatt T, et al.: Brief assessment of subjective health complaints: development, validation and population norms of a brief form of the Giessen Subjective Complaints List (GBB-8). J Psychosom Res. 2017, 95:33-43. 10.1016/j.jpsychores.2017.02.003

11. Gierk B, Kohlmann S, Kroenke K, et al.: The somatic symptom scale-8 (SSS- 8): a brief measure of somatic symptom burden. JAMA Intern Med. 2014, 174:399-407. 10.1001/jamainternmed.2013.12179

12. Wang J, Guo WJ, Mo LL, et al.: Prevalence and strong association of high somatic symptom severity with depression and anxiety in a Chinese inpatient population. Asia Pac Psychiatry. 2017, 9:12282. 10.1111/appy. 12282

13. Zilz C, Blaas SH, Pfeifer M, Jörres RA, Budweiser S: Mental health, serum biomarkers and survival in severe COPD: a pilot study. Multidiscip Respir Med. 2016, 11:3. 10.1186/s40248-016-0041-8

14. Serber ER, Edwards-Hampton SA, Yeager B, et al.: Prevalence of chest pain, depression, somatisation, anxiety, global distress, and substance use among cardiac and pulmonary rehabilitation patients. Pain Res Treat. 2012, 2012:138680. 10.1155/2012/138680

15. Zonneveld LN, Sprangers MA, Kooiman CG, Van 't Spijker A, Busschbach JJ: Patients with unexplained physical symptoms have poorer quality of life and higher costs than other patient groups: a cross-sectional study on burden. BMC Health Serv Res. 2013, 10:520. 10.1186/1472-6963-13-520

16. Barsky AJ, Peekna HM, Borus JF: Somatic symptom reporting in women and men . J Gen Intern Med. 2001, 16:266-75. 10.1046/j.1525-1497.2001.016004266.x

17. Slavney PR, Teitelbaum ML: Patients with medically unexplained symptoms: DSM-III diagnoses and demographic characteristics. Gen Hosp Psychiatry. 1985, 7:21-5. 10.1016/0163-8343(85)90006-4

18. Koola MM, Kuttichira P: Psychosocioeconomic study of medically unexplained physical symptoms . Indian J Psychol Med. 2012, 34:159-63.

19. Dirkzwager AJE, Verhaak PFM: Patients with persistent medically unexplained symptoms in general practice: characteristics and quality of care. BMC Fam Pract. 2007, 8:33. 10.1186/1471-2296-8-33

20. Coast E, Leone T, Hirose A, Jones E: Poverty and postnatal depression: a systematic mapping of the evidence from low and lower middle income countries. Health Place. 2012, 18:1188-97. 10.1016/j.healthplace.2012.05.004

21. Lund C, Breen A, Flisher AJ, et al.: Poverty and common mental disorders in low and middle income countries: a systematic review. Soc Sci Med. 2010, 71:517-28. 10.1016/j.socscimed.2010.04.027

22. Das J, Do QT, Friedman J, McKenzie D, Scott K: Mental health and poverty in developing countries: revisiting the relationship. Soc Sci Med. 2007, 65:467-80. 10.1016/j.socscimed.2007.02.037

23. Halpern-Manners A, Schnabel L, Hernandez EM, Silberg JL, Eaves LJ: The relationship between education and mental health: new evidence from a discordant twin study. Soc Forces. 2016, 95:107-31. 10.1093/sf/sow035

24. Kashyap A, Chopra S, Ranjan P, Sarkar S, Poorna A, Baitha U: Yoga therapy for medically unexplained physical symptoms. Adv Mind Body Med. 2020, 34:17-21. 